\title{
Potencial energético de clones de Paulownia y Miscanthus para la producción de energía en Chile
}

\author{
Energy potential of Paulownia and Miscanthus clones for energy \\ production in Chile
}

\author{
Hector Pegoretti* ${ }^{* 1}$,Fernando Muñoz', Regis Teixeiral,2, Katia Sáez', Cristina Segura ${ }^{3}$ y Rodrigo Olave ${ }^{4}$
}

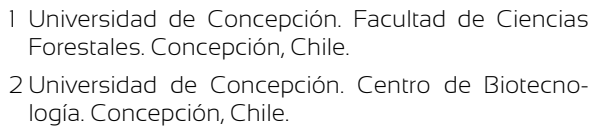

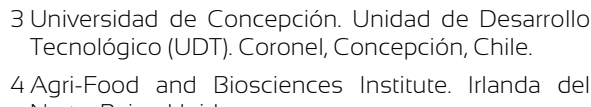
* Autor de correspondencia. hectorj.leite@gmail.com

\section{RESUMEN}

El modelo energético mundial se basa en combustibles fósiles, el cual ha venido siendo sustituido por consideraciones ambientales y económicas, dándole paso a la biomasa y a las plantaciones dendroenegéticas. En Chile, las energías renovables no convencionales (ERNC) vienen ganando espacio, además la nación ha abocado esfuerzos en especies que posean buen potencial energético. Así, este estudio tiene como objetivo evaluar el potencial físico-químico, energético y perfiles térmicos de dos especies (Paulownia elongata x fortunei y Miscantbus $\times$ giganteus) para el uso energético en tres sitios contrastantes de Chile. Los análisis fueron realizados en su mayoría bajo los estándares de las Normas DIN. Los resultados para Paulownia mostraron diferencias entre los sitios, obteniendo los mejores resultados en el sitio El Vergel con un porcentaje de lignina de 34,84\%, cenizas de 1,16\%, material volátil de 81,70\%, carbono de 47,02\% y una densidad energética líquida (DEL) de 2,16 GJ m³. Para Miscanthus los mejores resultados también fueron registrados en El Vergel, con porcentaje de lignina de $30,60 \%$, cenizas de $2,84 \%$, material volátil de $82,26 \%$ y carbono de $45,97 \%$ y una DEL de $1,67 \mathrm{GJ} \mathrm{m}^{-3}$, destacando que los mejores resultados de El Vergel son para la densidad D1. Respecto al perfil térmico (TGA-DTG), la Paulownia presentó pérdida de masa mayor que el Miscanthus y tuvo ignición a menor temperatura de $197,76{ }^{\circ} \mathrm{C}$ y $209,66{ }^{\circ} \mathrm{C}$ respectivamente. Por lo tanto, el mayor porcentaje de residuo lo presentó el Miscanthus con 5,63\%. Consiguientemente, ambas especies presentan características deseables para su uso energético.

PALABRAS CLAVE: análisis térmico, bioenergía, biomasa alternativa, energía renovable, ignición, TGA.

\section{ABSTRACT}

The global energy model is based on fossil fuels, which has been replaced by environmental and economic considerations, giving way to biomass and dendroenegetic plantations. In Chile, non-conventional renewable energies (NCRE) have been gaining space, and the nation has focused efforts on species with good energy potential. Thus, this study aims to evaluate the physical-chemical potential, energy and thermal profiles of two species (Paulownia elongata $\mathrm{x}$ fortunei and Miscanthus $\times$ giganteus) for energy use in three contrasting sites of Chile. The analyses were mostly performed under DIN Standards. The results for Paulownia showed differences between the sites, obtaining the best results in the Vergel site with a percentage of lignin of 34,84\%, ashes of 1,16\%, volatile material of $81.70 \%$, carbon of $47.02 \%$ and a liquid energy density (EL) of $2.16 \mathrm{GJ} \mathrm{m}^{-3}$. For Miscantbus, the best results were also recorded in the Vergel, with a percentage of lignin of $30.60 \%$, ashes of $2.84 \%$, volatile material of $82.26 \%$ and carbon of $45.97 \%$ and a DEL of $1,67 \mathrm{GJ} \mathrm{m}^{-3}$, highlighting that the best results of the Vergel site are for density D1. With respect to the thermal profile (TGA-DTG), Paulownia presented mass loss greater than Miscanthus and had ignition at a lower temperature of $197.76{ }^{\circ} \mathrm{C}$ and $209.66^{\circ} \mathrm{C}$ respectively. Therefore, the highest percentage of waste was presented by Miscanthus with 5.63\%. Accordingly, both species have desirable characteristics for their energetic use.

KEYWORDS: thermal analysis, bioenergy, alternative biomass, renewable energy, ignition, TGA. 


\section{INTRODUCCIÓN}

En Chile la generación de energía eléctrica a partir de fuentes renovables aporta alrededor del $11,74 \%$ a la matriz energética total (Centro Nacional para la Innovación y Fomento de las Energías Sustentables [CIFES], 2016). El Estado de Chile se ha venido planteando el desafío de promover el incremento de este tipo de energía, a través del desarrollo de estrategias enmarcadas en la reducción de emisiones provenientes de combustibles fósiles, comprometiéndose de esta forma con el incremento de las energías renovables y el fomento a la biomasa forestal con fines energéticos (Tolosana, 2009). Todo esto con el objetivo de fomentar soluciones dirigidas a impulsar la bioenergía en la matriz energética nacional. En este sentido, una de las alternativas para aumentar la producción de energía renovable es incentivar el establecimiento de cultivos dendroenergéticos, que posibiliten la producción y utilización de biomasa, así como también, la creación de oportunidades para reducir el abandono gradual de cultivos tradicionales.

Una especie que posee excelentes características y que además se muestra favorable para la producción de biomasa, es Miscanthus × giganteus J. M. Greef \& Deuter ex Hodk. \& Renvoize., la cual es una hierba perenne C4 que se caracteriza por una alta absorción de $\mathrm{CO}_{2}$, alto potencial de rendimiento y bajo consumo de agua (Płażek y Dubert, 2010). También ha sido ampliamente cultivada como fuente de bioenergía por más de 30 años (Scally, Hodkinson y Jones, 2007).

Asimismo, Miscanthus x giganteus tiene buena capacidad de adaptación a diferentes condiciones de suelo, es decir, desde arena a suelos de arcilla con alto contenido de materia orgánica, como también en suelos pobres. El-Bassam (1996) señala que los mejores rendimientos se muestran en suelos profundos, con alto contenido de materia orgánica, alta capacidad de retención de agua y buen drenaje. Otro estudio realizado en la zona centro sur de Chile reveló que, en sectores costeros de suelos de textura franco-arcillosalimosa con capacidad de retención de humedad, se obtuvo mejor rendimiento en biomasa (Muñoz y Cancino, 2014a).
La especie Miscanthus sp ha adquirido gran relevancia en el sector productivo, especialmente en Europa. Se ha demostrado que la biomasa de Miscanthus x giganteus puede ser utilizada como fuente de combustible sólido, así como también en la fabricación de materiales de construcción y como fuente de celulosa (Lewandowski, Clifton-Brown, Scurlocky Huisman, 2000). Sin embargo, su principal uso es la producción de energía, siendo considerada una excelente especie para estos fines. Otra especie de interés para la generación de energía es Paulownia sp también conocidas como kiri. Considerado como el árbol del futuro se han venido expandiendo su cultivo con fines maderables y energéticos. Posee tolerancia a diferentes condiciones de suelo y clima (Wang y Shogren, 1992), incluyendo amplios rangos de temperatura y precipitación, inclusive presenta un buen desempeño en suelos pobres (Kasamaki, 2007).

Además, el género posee una alta capacidad de crecimiento en ciclos cortos de producción, generando alta cantidad de biomasa para energía (López, Pérez, Zamudio, De Alva y García, 2012). Todas las especies y clones de este género son similares en apariencia y propiedades de la madera. Sin embargo, el clon Paulownia eloganta $\times$ fortunei se diferencia por corresponder a un híbrido natural que combina en su genotipo las características de dos especies destacadas por su crecimiento, adaptabilidad a diferentes tipos de sitios, resistencia a la sequía y a las bajas temperaturas, ideal para el uso energético (Hua, Ching-Ju, Xin-Yu y Yao Gao, 1986).

Por otro lado, la generación de energía a partir de la biomasa lignocelulósica promueve la descomposición térmica de los componentes químicos de la biomasa, la cual puede ocurrir mediante un proceso de carbonización sobre atmósfera inerte o en una atmósfera oxidante. Las fracciones de los componentes de las biomasas tiene una cinética de descomposición térmica bien diferenciada, por lo tanto, es bien conocido que las hemicelulosas sufren los mayores vértices de degradación entre $200{ }^{\circ} \mathrm{C}-300^{\circ} \mathrm{C}$, la celulosa entre $240{ }^{\circ} \mathrm{C}-350{ }^{\circ} \mathrm{C}$ y la lignina oscila entre 350 ${ }^{\circ} \mathrm{C}-500{ }^{\circ} \mathrm{C}$. Por lo tanto, es necesario evaluar el perfil térmico mediante técnicas que faciliten la comprensión del proceso. En este contexto, el análisis termogravimétrico 
(TGA) puede ser utilizado para determinar reacciones de descomposición y análisis de estabilidad térmica (Zattera, Bianchi, Zeni y Ferreira, 2005). Este método nos permite determinar la degradación térmica de la biomasa, observándose cuando inicia y donde es más acentuada, también nos suministra el porcentaje de residuos que se produce a una temperatura determinada. Por esta razón es una herramienta relevante a la hora de seleccionar especies para fines energéticos. Estos aspectos se han convertido en factores importantes para decisiones de establecimiento de este tipo de cultivos en el territorio nacional. Un ejemplo de esto, han sido los estudios desarrollados en la región de Antofagasta durante el año 2010, con el objetivo de establecer ensayos para obtener biomasa para energía (Muñoz y Cancino, 2014b).

Una investigación de Fernández-Puratich, OliverVillanueva, Mireya, Salvador y Nuria, (2014) muestra el porcentaje de biomasa de Paulownia de 13,9 t ha-1 y 15,3 t $\mathrm{ha}^{-1}$, que es relativamente alta, a pesar que su fuste es hueco en su sección medular. También posee alto poder calorífico que oscila entre $16 \mathrm{MJ} \mathrm{kg}^{-1}$ y $19 \mathrm{MJ} \mathrm{kg}^{-1}$, siendo en ocasiones mejor que algunas especies forestales que son utilizadas en la producción de bioenergía. Miscantbus es una especie bastante utilizada generalmente para la producción de etanol en la mayoría de los países, dando resultados positivos; por ejemplo un caso registrado por la Facultad de Agronomía de la Universidad de Buenos Aires [FAUBA] (2012), demuestra que el Miscanthus, a pesar que rinde menos en megagramos por hectárea, su producción de etanol es mayor que otras especies oscilando entre $10000 \mathrm{~L}$ $\mathrm{ha}^{-1}$ y $12000 \mathrm{~L} \mathrm{ha}^{-1}$ utilizadas para este fin como es el caso de maíz y caña de azúcar principalmente.

A diferencia de otros países pertenecientes a la región europea, en Chile no ha habido experiencia en la producción de biomasa para fines de uso energético con ambas especies mencionadas, y a su vez, existe escasa evidencia científica hasta el momento que permita sustentar el futuro establecimiento masivo de plantaciones productivas dirigidas a fines energéticos (FernándezPuratich et al., 2014; Muñoz, Cancino, Rodríguez y Olave, 2018). Por lo tanto, la presente investigación consistió en evaluar las características físicas, químicas y energéticas de clones de Miscanthus $\times$ giganteus y Paulownia eloganta $\times$ fortunei establecidos en tres suelos contrastantes del centro sur de Chile a través de los siguientes objetivos.

\section{OBJETIVOS}

El objetivo principal fue evaluar el potencial energético de clones de Paulownia y Miscanthus en tres sitios contrastantes y como objetivos específicos: a) determinar las características físico-químicas de las especies; b) evaluar las propiedades energéticas para la producción de estas especies con fines energéticos; c) analizar la degradación térmica de las biomasas y finalmente d) determinar el mejor sitio para el plantío de ambas especies.

\section{MATERIALES Y MÉTODOS}

\section{Biomasa}

Para evaluar su potencial se establecieron ensayos dendroenergéticos con Miscanthus × giganteus y Paulownia elongata $\times$ fortunei, en tres sitios contrastantes de la zona centro sur de Chile (Muñoz y Cancino, 2014b). Con este estudio se dio paso al desarrollo de nuevas oportunidades de producción de biomasa que aporten a la matriz energética del país. El material (clones) utilizado proviene de un proyecto que se llevó a cabo entre los años 2010 y 2014. Se establecieron en tres localidades, el primero fue el fundo El Vergel (Chaimávida, Concepción). El segundo sitio corresponde al fundo La Isla (Quillón) de típico clima mediterráneo templado cálido. Su morfología es ondulada a plana, se caracteriza por tener suelos derivados de rocas metamórficas. Por lo tanto, posee bajo porcentaje de fosforo, nitrógeno y materia orgánica, lo cual dificulta el establecimiento de cultivos agrícolas mas no de la acción forestal. Por último, el tercer sitio corresponde al fundo Trehualemu (a 13 kilómetros de Buchupureo), ubicado en la Región del Maule, pertenece a la franja costera ubicada entre la vertiente occidental de la costa y el Océano Pacifico. La ubicación e información edafoclimática de los tres sitios está ilustrada en la tabla 1. 
TABLA 1. Ubicación de los ensayos de Paulonia elongata $x$ fortunei y Miscanthus × giganteus

\begin{tabular}{|c|c|c|c|c|}
\hline Sitio & Coordenadas & $\begin{array}{l}\text { Precipitación } \\
\text { media anual }\end{array}$ & Clima & Suelo \\
\hline $\begin{array}{l}\text { Trehualemu } \\
\text { Región VII }\end{array}$ & $\begin{array}{l}\text { Latitud sur: } 35^{\circ} 58^{\prime} 29^{\prime \prime} \\
\text { Longitud este: } 72^{\circ} 45^{\prime} 8^{\prime \prime}\end{array}$ & $890 \mathrm{~mm}$ & $\begin{array}{l}\text { Agroclimática } \\
\text { secano costero }\end{array}$ & $\begin{array}{l}\text { Franco arcillo limosa } \\
\text { (suelos profundos) }\end{array}$ \\
\hline $\begin{array}{l}\text { El Vergel } \\
\text { Región VIII }\end{array}$ & $\begin{array}{l}\text { Latitud sur: } 36^{\circ} 50^{\prime} 30^{\prime \prime} \\
\text { Longitud oeste: } 72^{\circ} 54^{\prime} 26^{\prime \prime}\end{array}$ & $1200 \mathrm{~mm}$ & $\begin{array}{l}\text { Agroclimática } \\
\text { secano costero }\end{array}$ & $\begin{array}{c}\text { Franco arcillo limosa } \\
\text { (moderadamente } \\
\text { profundos) }\end{array}$ \\
\hline
\end{tabular}

El material de Miscanthus proviene de dos densidades (D1 $=20000$ brotes $\mathrm{ha}^{-1} ; \mathrm{D} 2=40000$ brotes $\mathrm{ha}^{-1}$ ) recolectados en los tres sitios (Tabla 1), la biomasa fue triturada y homogenizada por completo (caña, ramas y hojas), posteriormente fue tamizada entre las mallas 40 y 60 , utilizando el material retenido en 60 para los análisis y el restante fue considerado como polvo (utilizado para TGA). Por lo tanto, se obtuvo una muestra compuesta (mezcla) en cada sitio y para cada densidad, para así determinar sus características físico-químicas y energéticas. Para Paulownia, el material recolectado proviene de una sola densidad (D0 $=1000$ arb ha $^{-1}$ ) plantada en las mismas tres localidades, se realizó el mismo procedimiento que el Miscanthus, salvo que se utilizó solamente el fuste para esta especie. El experimento consistió en 15 tratamientos con tres repeticiones para cada parámetro evaluado. Los diferentes tratamientos de las biomasas estudiadas están ilustrados en la tabla 2.

\section{Caracterización física, química y energética de la biomasa}

La humedad (base seca) de la biomasa se obtuvo a través de la metodología descrita por la norma DIN EN 14774-1 (Deutsches Institut Für Normung [DIN], 2010a). La densidad a granel fue realizada de acuerdo con la norma DIN EN 15103 (DIN, 2010c). Para la determinación de la composición química y el poder calorífico superior de las muestras, se utilizaron muestras molidas en un molino de laboratorio tipo Wiley, de acuerdo con la norma TAPPI T 257 cm-85 (Technical Association of the Pulp and Paper Industry [TAPP], 1985).

La caracterización de la composición química de la biomasa consideró el contenido de extractivos utilizando la norma TAPPI T 204 cm-97 (TAPPI, 1997), utilizando el método de extractivos totales, el cual fue realizado con acetona $90 \%$. El contenido de lignina fue realizado por hidrolisis ácida de acuerdo con Mendonça, Jara, González, Elissetche y Freer (2008), quienes colectaron y filtraron el material residual a través de un gooch número 3, los sólidos fueron secados a una temperatura de $105^{\circ} \mathrm{C}$ hasta obtener su peso constante y fue determinado como lignina insoluble. La lignina soluble fue determinada por la medición de la absorbancia de la solución a $205 \mathrm{~nm}$.

La determinación de la química inmediata de la biomasa, material volátil, cenizas y por diferencia el carbono fijo, fue mediante el procedimiento establecido de la norma ASTM D1762-84 (American Society for Testing Materials [ASTM], 2007). La composición de química elemental (carbono, hidrógeno, nitrógeno y azufre) fue determinada en un equipo Vario Micro Cube CHNS-O, Elementar ${ }^{\circledR}$ y fue realizada de acuerdo con la norma DIN EN 15104 (DIN, 2011b). El valor de oxígeno fue determinado por la sumatoria del carbono, hidrógeno, nitrógeno, azufre y el contenido de cenizas decrecido de 100, de acuerdo con la norma DIN EN 15296 (DIN, 2011c). 
TABLA 2. Tratamientos, especies y origen de las especies utilizadas en el estudio

\begin{tabular}{|c|c|c|c|c|c|}
\hline Tratamiento & Especie & Sitio & Clon & $I D$ & Densidad de plantío \\
\hline $\mathrm{T} 1$ & Paulownia & La Isla & $\mathrm{Pl}$ & DO & 1000 arb ha-1 $^{-1}$ \\
\hline $\mathrm{T} 2$ & Paulownia & La Isla & $\mathrm{Pl}$ & DO & 1000 arb ha-1 \\
\hline T3 & Paulownia & La Isla & $\mathrm{Pl}$ & DO & 1000 arb ha-1 $^{-1}$ \\
\hline T4 & Paulownia & Trehualemu & $\mathrm{Pl}$ & DO & 1000 arb ha-1 $^{-1}$ \\
\hline T5 & Paulownia & Trehualemu & $\mathrm{Pl}$ & DO & 1000 arb ha-1 $^{-1}$ \\
\hline T6 & Paulownia & Trehualemu & $\mathrm{Pl}$ & DO & 1000 arb ha-1 $^{-1}$ \\
\hline $\mathrm{T7}$ & Paulownia & El Vergel & $\mathrm{Pl}$ & DO & 1000 arb ha-1 $^{-1}$ \\
\hline T8 & Paulownia & El Vergel & $\mathrm{Pl}$ & DO & 1000 arb ha-1 $^{-1}$ \\
\hline T9 & Paulownia & El Vergel & $\mathrm{Pl}$ & DO & 1000 arb ha-1 $^{-1}$ \\
\hline $\mathrm{T} 10$ & Miscanthus & Trehualemu & MP1 & $\mathrm{D} 1$ & 20000 brotes $\mathrm{ha}^{-1}$ \\
\hline $\mathrm{T} 11$ & Miscanthus & Trehualemu & MP2 & $\mathrm{D} 2$ & 40000 brotes ha-1 \\
\hline $\mathrm{T} 12$ & Miscanthus & La Isla & MP1 & $\mathrm{D} 1$ & 20000 brotes ha-1 \\
\hline $\mathrm{T} 13$ & Miscanthus & La Isla & MP2 & D2 & 40000 brotes ha ${ }^{-1}$ \\
\hline $\mathrm{T} 14$ & Miscanthus & El Vergel & MP1 & $\mathrm{D} 1$ & 20000 brotes $\mathrm{ha}^{-1}$ \\
\hline $\mathrm{T} 15$ & Miscanthus & El Vergel & MP2 & $\mathrm{D} 2$ & 40000 brotes ha-1 \\
\hline
\end{tabular}

Para determinar el poder calorífico superior (PCS), se utilizó una bomba calorimétrica modelo PARR 6400 mediante el procedimiento de la norma DIN EN 14918 (DIN, 2010b). El poder calorífico inferior (PCI) fue estimado mediante la ecuación (1); donde ambos son calculados en $\mathrm{kcal} \mathrm{kg}^{-1} \mathrm{y} \mathrm{H}$ es el contenido de hidrógeno (\% en base seca), de acuerdo con (Brand, 2010).

$$
\text { PCI }=\text { PCS }-(600 \times 9 H) / 100
$$

La estimación del poder calorífico líquido o útil (PCL), fue realizada utilizando la ecuación (2); conforme al anexo E de la norma DIN EN 14918 (DIN, 2010b)

$$
\mathrm{PCL}=\mathrm{PCI} \times\left[\left(\frac{100-\mathrm{U}_{\mathrm{bu}}}{100}\right)\right]-\left(6 \times \mathrm{U}_{\mathrm{bu}}\right)
$$

Donde:

$\mathrm{U}_{\mathrm{bu}}=$ humedad base húmeda.
De esta forma fue posible calcular la densidad energética a granel de las biomasas, mediante la ecuación (3);

$$
\mathrm{DE}_{\mathrm{G}}=\mathrm{DG} \times \mathrm{PCL}
$$

Donde:

$\mathrm{DE}_{\mathrm{G}}=$ densidad energética a granel

$\mathrm{DG}=$ densidad a granel

\section{Análisis termogravimétrico}

En este trabajo se analizó la reactividad mediante el criterio termogravimétrico por temperatura. 1) La temperatura de base ( $\left.T_{\text {base }}\right)$ o temperatura de ignición es el punto donde se obtiene un valor de la derivada DTG = $1\left(\%\right.$ mas $\left.\min ^{1}\right)$; donde las muestras más reactivas corresponderán a las que alcanzan el valor base (DTG) a menores temperaturas ( $\left.T_{\text {base }}\right)$ y menor tiempo, esto se debe a la liberación de los volátiles más fácilmente (Ghetti, 1986). 2) La temperatura 
en la que se da la máxima tasa de pérdida de masa durante la etapa de devolatilización $\left(T_{\max }\right)$. Esta variable se considera para verificar si existe una relación entre análisis próximo y el análisis térmico de las muestras.

Para el análisis de termogravimétrico (TGA), se utilizaron muestras de biomasas seca y molidas $(5 \mathrm{mg})$ por triplicado empleando un equipo TGA/DSC1 (Mettler Toledo, Switzerland), llegando a una temperatura de $600{ }^{\circ} \mathrm{C}$ en atmósfera de aire con una velocidad de aumento de temperatura de $20{ }^{\circ} \mathrm{C}$ min $^{-1}$ para evaluar la pérdida máxima de peso.

\section{Análisis estadísticos}

Para cada propiedad cuantificada de las especies, fueron calculadas las tendencias centrales de media y dispersión (desviación estándar). Para evaluar si existen diferencias estadísticas significativas entre los sitios o entre densidades se realizó un análisis de delineamiento enteramente casualizado (DIC), luego se aplicó una comparación de medias a través de la prueba de Tukey con 5\% de probabilidad. Se empleó el software estadístico SPSS (versión 24.0). Posteriormente se realizó un análisis multivariado de componentes principales (PCA), para así reducir la dimensión del conjunto de datos, conservando la variación de datos. El cual permitió verificar la influencia de cada variable y su correlación.

\section{RESULTADOS Y DISCUSIÓN}

\section{Características físicas, químicas y energéticas de Paulownia elongata $x$ fortunei}

Los resultados obtenidos de la caracterización química inmediata (lignina, extractivos, cenizas, carbono fijo y volátiles), química elemental (CHNS-O) y los poderes caloríficos (PCI, PCS, PCL y DEL) de la Paulownia elongata $x$ fortunei son ilustrados en la tabla 3 , los valores muestran las comparaciones entre sitios. La lignina es un componente importante y deseable para el área energética, ya que contribuye al aumento de los poderes caloríficos de las biomasas (Demirbas, 2002). Los resultados obtenidos arrojaron un promedio de lignina de $34,41 \%$, destacando que estos valores son similares a los encontrados por Muñoz y Cancino (2014b). Caso contrario a los valores de lignina hallados por (Kalaycioglu, Deniz, y Hiziroglu, 2005), quienes registraron valores de $22,1 \%$, lo cual es un punto positivo visando la producción dendroenergética. Esto nos indica que es factible plantar la especie en los tres sitos para fines energéticos (valores encontrados favorables), ya que es de conocimiento que el sitio puede afectar la composición química debido a las propiedades o nutrientes existes en cada lugar.

Por otra parte, respecto a los extractivos, autores como (Vargas-moreno, Callejón-ferre, Pérez-alonso y VelázquezMartí, 2012), definen que estos desempeñan un papel importante en el uso de las biomasas como fuente de energía, y que además, contribuyen a elevar el poder calorífico de las biomasas junto con la lignina. En este sentido, en el presente estudio se observó una media de extractivos de 9,29\%, donde no hubo diferencia significativa entre los sitios, estos valores también son similares a los encontrados por Muñoz y Cancino (2014b). Componentes como la lignina y los extractivos son parámetros importantes en la parte energética, ya que bajos porcentajes en ambas cuantificaciones podría ser un factor para descartar una especie en la producción de bioenergía, debido a que disminuiría el poder calorífico.

Respecto a las variaciones del carbono fijo, que es la cantidad de carbono retenida en la biomasa después de perder todas las sustancias que volatizaron en el proceso y que no es ceniza, pueden ser explicadas por las diferencias de los porcentajes de lignina y extractivos, ya que, a mayor cantidad de lignina mayor porcentaje de carbono fijo (Demirbaş, 2003). Referente a lo anterior, la media obtenida en este estudio para este parámetro fue de $18,29 \%$, y no se presentaron diferencias significativas entre sitios. Esto señala que combustibles que posean mayores valores de carbono fijo se quemarán o consumirán más lentamente, ya que es una fracción no volátil que se oxida en el estado sólido. Estos valores obtenidos son superiores al ser comparados con el estudio realizado por Pinto (2015), con Paulownia tomentosa y Paulownia elongata $\times$ fortunei, donde la 
media fue de 13,26\%. De acuerdo con (Poddar, Kamruzzaman, Sujan, Hossain, Jamal y Gafur, 2014), los materiales volátiles contribuyen a la ignición del combustible. En este estudio se obtuvo una media de 80,38\% y no presentó diferencias significativas entre sitios. Este porcentaje resultó bajo respecto a valores hallados por Guofeng (2014), quien registró volátiles de $P$. tomentosa y $P$. elongata de 85,03\% y 81,68\% respectivamente. Esto sería una desventaja, ya que altos valores de materiales volátiles son favorables para la ignición y el uso energético.

Por otra parte, la química elemental (carbono, hidrógeno, nitrógeno, azufre y oxígeno) de la biomasa en la producción de bioenergía es relevante. Es deseable que la biomasa presente altos contenidos de carbono e hidrógeno y bajos valores de oxígeno y cenizas (Paula, Trugilho, Napoli y Bianchi, 2011), para tener un mejor rendimiento y aprovechamiento del combustible. En la tabla 3 se ilustran los resultados de la química elemental, donde se observa que la media del carbono e hidrógeno es de 45,88\% y $6,35 \%$, respectivamente, estos valores son similares a los encontrados por Fernández-Puratich et al. (2014). También obtener alto porcentaje de carbono e hidrogeno es importante, ya que contribuyen con un mayor equilibrio en la liberación de energía y en la formación de $\mathrm{CO}_{2}$ y $\mathrm{H}_{2} \mathrm{O}$ (Obernberger y Thek, 2010). Respecto al nitrógeno en los tres sitios evaluados se registraron valores con media de $1,04 \%$, es decir, el doble de lo que se puede encontrar en la literatura (alrededor de 0,5\%) (Obernberger, Brunner y Barnthaler, 2006). De acuerdo con los mismos autores, porcentajes superiores a $0,6 \%$ causan impactos negativos al ambiente debido a la liberación de óxidos nítricos durante la combustión.

TABLA 3. Resultados de la caracterización de la Paulownia elongata $\times$ fortunei

\begin{tabular}{|c|c|c|c|c|c|c|c|c|}
\hline \multirow[b]{2}{*}{ Variable } & \multicolumn{2}{|c|}{ Isla } & \multicolumn{2}{|c|}{ Trehualemu } & \multicolumn{2}{|c|}{ Vergel } & \multirow[b]{2}{*}{ R2 } & \multirow[b]{2}{*}{ Valor-p } \\
\hline & Media & $D . E$. & Media & D.E. & Media & $D . E$. & & \\
\hline Lignina (\%) & $32,98^{b}$ & 1,96 & $35,42^{a}$ & 1,563 & $34,84^{a b}$ & 1,58 & 0,35 & 0,266 \\
\hline Extraíbles (\%) & $8,86^{a}$ & 1,37 & $9,14^{\text {a }}$ & 0,709 & $9,85^{a}$ & 2,17 & 0,09 & 0,732 \\
\hline Cenizas (\%) & $1,19^{b}$ & 0,41 & $1,62^{a}$ & 0,079 & $1,16^{b}$ & 0,03 & 0,52 & 0,106 \\
\hline Carbono fijo (\%) & $18,63^{a}$ & 1,85 & $19,24^{\mathrm{a}}$ & 1,785 & $17,18^{a}$ & 0,13 & 0,33 & 0,290 \\
\hline Volátiles (\%) & $80,20^{a b}$ & 1,67 & $79,21^{\mathrm{b}}$ & 1,898 & $81,62^{a}$ & 0,14 & 0,40 & 0,208 \\
\hline Carbono (\%) & $45,44 \mathrm{ab}$ & 0,49 & $45,12^{b}$ & 1,053 & $46,84^{a}$ & 1,44 & 0,42 & 0,193 \\
\hline Hidrogeno (\%) & $6,11^{\text {a }}$ & 0,19 & $6,36^{a}$ & 0,133 & $6,49^{a}$ & 0,26 & 0,47 & 0,148 \\
\hline Nitrógeno (\%) & $1,12^{a}$ & 0,09 & $1,16^{a}$ & 0,299 & $0,95^{a}$ & 0,05 & 0,28 & 0,372 \\
\hline Azufre (\%) & $0,32^{a}$ & 0,05 & $0,20^{b}$ & 0,058 & 0,27 ab & 0,01 & 0,65 & 0,041 \\
\hline Oxígeno (\%) & 45,59 a & 0,61 & $45,69^{a}$ & 1,433 & $44,09^{a}$ & 1,94 & 0,27 & 0,373 \\
\hline H. (base seca) (\%) & $6,16^{b}$ & 0,15 & $6,72^{a}$ & 0,21 & 6,59 ab & 0,31 & 0,34 & 0,455 \\
\hline Dens. granel $\left(\mathrm{kg} \mathrm{m}^{-3}\right)$ & $170,52^{a}$ & 0,18 & $124,52^{a}$ & 0,46 & $144,25^{a}$ & 0,32 & 0,75 & 0,421 \\
\hline $\mathrm{PCl}\left(\mathrm{MJ} \mathrm{kg}^{-1}\right)$ & $16,35^{\text {a }}$ & 0,17 & $16,15^{a b}$ & 0,071 & $16,08^{b}$ & 0,15 & 0,49 & 0,125 \\
\hline PCS (MJ kg-1) & $17,70^{a}$ & 0,17 & $17,51^{\mathrm{ab}}$ & 0,071 & $17,44^{a}$ & 0,15 & 0,49 & 0,125 \\
\hline PCL (MJ kg-1) & $15,35^{\text {a }}$ & 0,57 & $14,98 \mathrm{ab}$ & 0,210 & $14,93^{b}$ & 0,21 & 0,26 & 0,389 \\
\hline DEL (GJ $\mathrm{m}^{-3}$ ) & $2,62^{a}$ & 0,18 & $1,86^{c}$ & 0,020 & $2,15^{b}$ & 0,03 & 0,92 & 0,000 \\
\hline
\end{tabular}

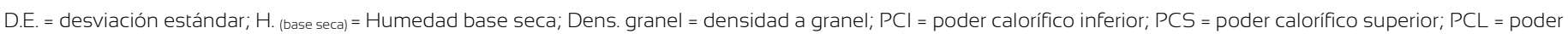
calorífico liquido; DEL = densidad energética liquida; Medias iguales por la misma letra no difieren entre sí, a 1\% de probabilidad por la prueba de Tukey. 
Por otra parte, valores de azufre superiores a 0,1\% están relacionados a corrosión de los equipos (estufas) utilizadas para la combustión de la biomasa, esto es debido a la formación de sales alcalinas (Obernberger et al., 2006). Entre los tres sitios evaluados, La Isla presentó el mayor valor de azufre entre los sitios, registrando una media de 0,25\%. Este valor es alto respecto al hallado por Fernández-Puratich et al. (2014), quienes registraron un valor de azufre de $0,03 \%$ en Paulownia spp. Estos altos valores de azufre registrados en los sitios se deben al efecto aerosol del mar que es una fuente importante de azufre; por lo tanto, sitios ubicados cerca del litoral costero pueden recibir importante aporte de este elemento. Por otro lado, el oxígeno también es un inconveniente ya que elevados porcentajes de nitrógeno y oxígeno resultan en una disminución del valor calórico de la biomasa (Velázquez-martí, Sajdak, López-cortés y Callejónferre, 2014), así que lo valores de este estudio (40\% - 48\%) están dentro de los parámetros normales.

Los mayores valores energéticos provienen de biomasas que presenten altos porcentajes de lignina, mayor cantidad de resinas y aceites esenciales. Según Gillespie, Everard, Fagan y Mcdonnell (2013), señalan que el poder calorífico liquido PCL (o útil) y la densidad energética liquida DEL son parámetros importantes para poder caracterizar un material como combustible, ya que optimiza la cantidad de biomasa en la producción de energía, ayuda la parte logística y también se obtienen ideas para dimensionar las instalaciones donde será almacenado el material. Al respecto, en este estudio se observó que los poderes caloríficos (Tabla 3) de los tres sitios son similares a los estudios de Fernández-Puratich et al. (2014). Se observó que, en La Isla, la DEL fue mayor, esto se puede explicar debido a que también presentó el menor porcentaje de humedad, el cual favoreció a la DEL, caso contrario a lo sucedido en el sitio de Trehualemu. Por lo tanto, es posible determinar que la especie Paulownia elongata $\times$ fortunei se adaptó a los sitios evaluados, donde el conjunto de parámetros estudiados evidenció mejores resultados específicamente en el sitio descrito como el Vergel.
Características físicas, químicas y energéticas de Miscanthus $x$ giganteus

Es importante destacar, que las características de cada sitio pueden modificar ciertas propiedades del Miscanthus. Una de las variables importantes es la disponibilidad de agua que puede llegar a influenciar tanto el rendimiento (Roncucci, Nassi O Di Nasso, Bonari, y Ragaglini, 2015), como también los parámetros químicos y energéticos. Por lo tanto, tener conocimiento sobre la especie y el sitio es esencial a la hora del establecimiento de cualquier cultivo y más con fines energéticos.

Se evaluó el desempeño del Miscanthus $x$ giganteus plantados en los tres sitios ilustrados en la tabla 4, además se verificó la comparación de ambas densidades (D1-D2) en el mismo sitio como también entre los tres sitios. Se encontró 36,14\% de lignina en la D2 de La Isla (significativamente diferente a la densidad D1); esto demuestra el potencial de este sitio para el establecimiento de plantíos energéticos. Estos valores resultaron superiores a los obtenidos por (Muñoz y Cancino, 2014a), e inclusive superior a una especie de gramínea muy similar al Miscanthus y altamente utilizada con fines energético en Brasil como es el Capim-elefante (Pennisetum purpureum), el cual posee porcentajes de lignina de 23\% - 25\% (Pereira, 2014). En este sentido, al establecer comparaciones de cada densidad entre los tres sitios referente, es posible notar que la D2 de La Isla continuó destacándose sobre los demás sitios evaluados, aunque sólo con diferencias significativas con la densidad D2 del sitio Trehualemu.

El porcentaje de extractivos obtenidos en el sitio Trehualemu fue superior a los otros sitios con una media de 6,51\%. A su vez, los valores de extractivos de El Vergel son similares a los encontrados por Rosa, Hortal, Vidal, Colom y Torres (2004), en un estudio realizado en Miscanthus sinensis. Concerniente a la comparación de este parámetro entre los tres sitios, mostró una diferencia significativa ilustrado en la tabla 4. 
TABLA 4. Resultados de la caracterización del Miscanthus × giganteus

\begin{tabular}{|c|c|c|c|c|c|c|c|c|c|c|c|c|}
\hline \multirow{3}{*}{ Variable } & \multicolumn{4}{|c|}{ La Isla } & \multicolumn{4}{|c|}{ Trehualemu } & \multicolumn{4}{|c|}{ El Vergel } \\
\hline & \multicolumn{2}{|c|}{$D 1$} & \multicolumn{2}{|c|}{$D 2$} & \multicolumn{2}{|c|}{$D 1$} & \multicolumn{2}{|c|}{$D 2$} & \multicolumn{2}{|c|}{$D 1$} & \multicolumn{2}{|c|}{$D 2$} \\
\hline & Media & $D . E$. & Media & D.E. & Media & D.E. & Media & $D . E$. & Media & D.E. & Media & D.E. \\
\hline Lignina (\%) & 32,87 bc & 0,93 & $36,14^{a}$ & 0,44 & 34,01 ab & 0,51 & $30,37^{c}$ & 0,64 & $30,79^{c}$ & 1,52 & $30,42^{c}$ & 1,73 \\
\hline Extractivos (\%) & $5,77^{b}$ & 0,12 & $3,51^{c d}$ & 0,23 & $6,55^{a}$ & 0,29 & $6,48^{a}$ & 0,25 & $3,66^{c}$ & 0,29 & $3,01^{d}$ & 0,04 \\
\hline Cenizas (\%) & $6,02^{a}$ & 0,01 & $77,56^{b}$ & 0,30 & $4,28^{c}$ & 0,08 & $79,08^{d}$ & 0,26 & $2,67^{e}$ & 0,05 & $82,15^{d}$ & 0,29 \\
\hline Carbono fijo (\%) & $16,39^{b}$ & 0,30 & $17,31^{\text {a }}$ & 0,28 & $16,53^{b}$ & 0,30 & 16,79 ab & 0,18 & $15,24^{c}$ & 0,19 & $14,40^{d}$ & 0,23 \\
\hline Volátiles (\%) & $77,56^{d}$ & 0,30 & $76,87^{d}$ & 0,30 & $79,08^{c}$ & 0,26 & $80,47^{b}$ & 0,51 & $82,15^{a}$ & 0,29 & $82,37^{a}$ & 0,43 \\
\hline Carbono & $44,89 a b$ & 0,24 & $45,25^{a}$ & 0,13 & $43,18^{b}$ & 0,15 & $43,67^{c}$ & 0,18 & $47,57^{a}$ & 0,61 & $44,37^{b}$ & 0,17 \\
\hline Hidrogeno (\%) & $6,27^{c}$ & 0,03 & $6,09^{d}$ & 0,02 & $6,06^{d}$ & 0,05 & $6,48 a b$ & 0,04 & $6,36 \mathrm{bc}$ & 0,12 & $6,64^{a}$ & 0,06 \\
\hline Nitrógeno (\%) & $0,42^{a b}$ & 0,03 & $0,42^{a b}$ & 0,05 & $0,74^{a}$ & 0,05 & $0,52^{a}$ & 0,23 & $0,51^{a}$ & 0,15 & $0,13^{b}$ & 0,05 \\
\hline Azufre (\%) & $0,26^{a b c}$ & 0,02 & $0,26^{a b c}$ & 0,01 & $0,27^{a}$ & 0,01 & $0,23^{c}$ & 0,02 & $0,27^{a b}$ & 0,01 & $0,24^{b c}$ & 0,02 \\
\hline Oxígeno (\%) & $41,98^{c}$ & 0,49 & $42,09^{c}$ & 0,19 & $45,52 \mathrm{ab}$ & 0,07 & $45,83^{a}$ & 0,11 & $42,62^{b c}$ & 2,75 & $45,55^{a b}$ & 0,24 \\
\hline H, (base seca) & $8,25^{a}$ & 0,04 & $5,96^{b}$ & 0,02 & 6,29 a & 0,01 & $6,21^{a}$ & 0,07 & $6,60^{a}$ & 0,01 & $6,64^{a}$ & 0,04 \\
\hline Dens, granel & $117,38^{a}$ & 0,48 & $116,80^{b}$ & 0,36 & $114,47^{a}$ & 0,18 & $112,72^{b}$ & 0,31 & $113,68^{\text {a }}$ & 0,33 & 114,38 a & 0,37 \\
\hline $\mathrm{PCl}\left(\mathrm{MJ} \mathrm{kg} \mathrm{k}^{-1}\right)$ & $15,02^{a b}$ & 0,12 & $15,37^{a b}$ & 0,08 & $14,56^{b}$ & 0,13 & $14,57^{b}$ & 0,25 & $16,15^{a}$ & 1,07 & 15,47 ab & 0,24 \\
\hline PCS (MJ kg-1) & $16,37^{a b}$ & 0,12 & $16,73^{a b}$ & 0,08 & $15,92^{b}$ & 0,13 & $15,93^{b}$ & 0,25 & $17,50^{a}$ & 1,07 & $16,83^{a b}$ & 0,24 \\
\hline PCL (MJ kg-1) & $13,68^{b}$ & 0,11 & $14,37^{a b}$ & 0,08 & $13,55^{b}$ & 0,12 & $13,57^{b}$ & 0,24 & $14,99^{a}$ & 1,01 & 14,35 ab & 0,22 \\
\hline DEL (GJ m-3) & $1,61 \mathrm{abc}$ & 0,01 & $1,68 \mathrm{ab}$ & 0,01 & $1,55^{b c}$ & 0,01 & $1,53^{c}$ & 0,02 & $1,70^{a}$ & 0,12 & $1,64 a b c$ & 0,02 \\
\hline
\end{tabular}

$\mathrm{Dl}=20.000$ brotes ha-1; D2 = 40.000 brotes ha-1; D.E. = desviación estándar; $\mathrm{H}$. (base seca) = Humedad base seca; Dens. granel = densidad a granel; PCl = poder calorífico inferior; $\mathrm{PCS}=$ poder calorífico superior; PCL = poder calorííico liquido; $\mathrm{DEL}=$ densidad energética liquida; Medias iguales por la misma letra no difieren entre sí, a l\% de probabilidad por la prueba de Tukey. Todas las medias están expresadas en porcentaje \%. 
Referente al porcentaje de cenizas cuando se comparan ambas densidades en el mismo sitio, presentan diferencias significativas en ambas densidades. Los valores hallados en el estudio son superiores a los encontrados por Lino et al. (2014), donde se registró 1,90\% de cenizas en Miscanthus $\times$ giganteus. Por el contrario, en este estudio los valores para este parámetro son similares a resultados encontrados por el mismo autor en Miscanthus sinensis y Miscanthus floridulus con 4,36\% y 4,34\%, respectivamente. Ya cuando comparamos ambas densidades entre los tres sitios sobre dicho parámetro, podemos observar que la D1 es estadísticamente diferente entre los tres sitios. De igual forma los valores de este parámetro se encuadran dentro de los exigidos por las normas internacionales, como las EN PLUS, ISO y ÖNORM. Por lo cual es una especie cuyas densidades se adaptan a diferentes tipos de sitios y condiciones.

Acerca del carbono fijo varió entre 14,40\% - 17,31\%, destacándose como el mejor resultado el obtenido en la D2 de La Isla. Además, hubo una variación muy baja ya que los valores son muy similares, tanto cuando se comparan ambas densidades entre el mismo sitio como entre los tres locales. Asimismo, los resultados obtenidos son equivalentes a los encontrados por McKendry (2002), que señala valores de 15,9\% en Miscanthus spp. Por otra parte, el estudio realizado por Saldarriaga, Pablos, Amutio, Aguado y Olaza (2013) arrojó valores de 11,28\% en Miscanthus spp., los cuales son inferiores a los de este estudio. En este sentido, evaluar este parámetro es importante ya que tiene relación directa con la lignina, y ambos son resistentes a la descomposición térmica. Por lo tanto, los valores hallados en este estudio son deseables, ya que aumentará el poder calorífico de la biomasa.

Otro punto son los volátiles, el mayor porcentaje de material volátil lo presentó el sitio El Vergel, con una media de $82,26 \%$. Cuando comparamos ambas densidades entre sitios, se evidenciaron diferencias significativas. Estos resultados son significativamente mayores a los hallados por Santiago-Ortega, Honorato-Salazar, Quero-Carrillo, Hernandez-Garay, López-Castañeda y López-Guerrero (2016), quienes mencionan valores de 66,8\% en Miscanthus $s p p$. De acuerdo con otro estudio realizado por Saldarriaga et al. (2013), los resultados arrojaron 76,58\% de material volátil en Miscanthus spp., lo cual es similar a lo hallado en el sitio de La Isla, indicando que el material es de rápida ignición.

Respecto a la composición elemental, el porcentaje de carbono no tuvo diferencias significativas. Solo si es comparado entre sitios, la D2 presentó diferencias entre los sitios. En este sentido, lo encontrado en este estudio es similar al valor de 44,96\%, registrado por Delaye y Maia (2015) . Por otra parte, el valor de este estudio fue superior al registrado por Muñoz y Cancino (2014a), el cual fue de $41,90 \%$, en ambos estudios se evaluó Miscanthus spp. Esta diferencia puede atribuirse a las condiciones de cada sitio o al cambio de densidad del plantío.

En cuanto al hidrógeno y nitrógeno cuando comparamos las densidades (D1-D2) entre el mismo sitio y entre ellos son muy parecidas entre sí. Los resultados son semejantes a los señalados por Muñoz y Cancino (2014a). Algunos valores pueden ser menores o mayores, esto se debe a que no existe una estandarización para el cálculo, y estas cuantificaciones pueden ser en base seca como es el caso de este estudio, y a su vez, en base seca libre de cenizas.

En relación al azufre, los resultados fueron muy similares, destacando que las medias de todas las densidades y para todos los sitios fueron superiores al $0,1 \%$. Resultados equivalentes fueron registrados en el estudio de Muñoz y Cancino (2014a). Es importante destacar que cuando este valor es elevado, puede ser perjudicial tanto para el ambiente, así como también disminuye los valores energéticos de las biomasas. Otro factor indeseable son los altos valores de oxígeno debido a la combustión de la biomasa, ya que el carbono e hidrógeno contribuyen en mayor proporción en la liberación de energía, para formar $\mathrm{CO}_{2}$ y $\mathrm{H}_{2} \mathrm{O}$ (Obernberger y Thek, 2010). Por lo tanto, estos parámetros no presentaron diferencias significativas relevantes. Consiguiente, estos valores son aceptables y similares con otras biomasas no maderables.

Por otra parte, también se puede observar los valores físicos y energéticos de Miscanthus × giganteus en la tabla 4. En este sentido, es posible constatar que la humedad osciló 
entre $5 \%-10 \%$. Estos resultados atienden los parámetros exigidos por las normas internacionales (antes mencionadas) para fines energéticos. Respecto a la densidad a granel del material es un parámetro que no está estandarizado, pero nos da referencia de cómo será la densificación y si el producto final tendrá una buena compactación, durabilidad y resistencia mecánica para productos densificados.

Respecto a los valores energéticos, es importante destacar que hubo poca variabilidad en relación a sitio y densidad. Al respecto, el sitio donde se obtuvo mejores valores energéticos fue El Vergel, aunque presenta diferencias significativas muy bajas en comparación a los otros sitios. En cuanto al PCS, se registró una variación entre 15,92 MJ kg-1 - 17,50 MJ kg-1. Estos valores fueron similares a los resultantes en el estudio de Muñoz y Cancino (2014a), y a su vez, son inferiores a otros estudios realizados en Miscanthus y otros residuos agrícolas por Meehan, Finnan, yDonnell, 2013), donde se observaron valores superiores a $18,00 \mathrm{MJ} \mathrm{kg}^{-1}$.

Los bajos valores energéticos encontrados en este estudio pueden atribuirse a la humedad y al alto porcentaje de cenizas que se registró, ya que este afecta directamente los valores energéticos. De modo general los valores energéticos son menores a los de la Paulownia, debido a que las biomasas maderables poseen menores porcentajes de cenizas, los cuales ayudan a aumentar su poder calorífico, mientras que las biomasas herbáceas (Miscanthus × giganteus) poseen mayores porcentajes de cenizas los cuales son catalogados como indeseables. Otro aspecto importante de acuerdo con Baxter et al. (2014), es que la madera tiene más enlaces de carbono que las herbáceas, los cuales ayudan en los acrecimos energéticos.

\section{Análisis de los componentes principales (PCA)}

Se observó que las tres primeras componentes principales explican el $0,988 \%$ de la varianza total, a pesar de tener cuatro. Por lo tanto, las informaciones más notables de los datos originales están incluidas en esas tres componentes. En la tabla 5, se encuentran los autovectores y las correlaciones entre variables originales y los cuatro primeros componentes principales (CP1, CP2, CP3 y CP4) consideradas.

TABLA 5. Autovectores (e) y las correlaciones (r) entre las variables originales y las cuatro primeras componentes principales.

\begin{tabular}{|c|c|c|c|c|c|c|c|c|}
\hline \multirow{2}{*}{ Variables } & \multicolumn{2}{|c|}{$C P 1$} & \multicolumn{2}{|c|}{$C P 2$} & \multicolumn{2}{|c|}{ CP3 } & \multicolumn{2}{|c|}{ CP4 } \\
\hline & $e_{1}$ & $r$ & $e_{2}$ & $r$ & $e_{3}$ & $r$ & $e_{4}$ & $r$ \\
\hline DTG & 0,19 & 0,50 & 0,39 & 0,77 & $-0,29$ & $-0,34$ & 0,12 & 0,12 \\
\hline Shoulder & 0,11 & 0,28 & 0,41 & 0,81 & $-0,29$ & $-0,34$ & 0,31 & 0,29 \\
\hline Resíduo & 0,34 & 0,88 & 0,10 & 0,19 & $-0,03$ & $-0,03$ & 0,01 & 0,01 \\
\hline Extractivos (\%) & $-0,30$ & $-0,77$ & $-0,21$ & $-0,42$ & 0,11 & 0,13 & $-0,04$ & $-0,03$ \\
\hline Lignina (\%) & $-0,14$ & $-0,33$ & $-0,28$ & $-0,56$ & 0,30 & 0,35 & 0,40 & 0,38 \\
\hline Cenizas (\%) & 0,33 & 0,87 & 0,03 & 0,07 & $-0,22$ & $-0,26$ & 0,20 & 0,19 \\
\hline Volátiles (\%) & $-0,12$ & $-0,32$ & 0,42 & 0,83 & 0,23 & 0,27 & $-0,27$ & $-0,25$ \\
\hline C. fijo (\%) & $-0,22$ & $-0,57$ & $-0,24$ & $-0,46$ & $-0,38$ & $-0,45$ & 0,47 & 0,44 \\
\hline Carbono (\%) & $-0,17$ & $-0,45$ & 0,33 & 0,66 & 0,32 & 0,38 & 0,14 & 0,13 \\
\hline Hidrogeno (\%) & $-0,03$ & $-0,08$ & 0,25 & 0,50 & 0,49 & 0,57 & 0,46 & 0,43 \\
\hline Nitrógeno (\%) & $-0,30$ & $-0,79$ & $-0,07$ & $-0,14$ & $-0,23$ & $-0,28$ & 0,17 & 0,16 \\
\hline $\mathrm{PCl}(\mathrm{MJ} \mathrm{kg-1})$ & $-0,34$ & $-0,88$ & 0,22 & 0,43 & $-0,10$ & $-0,12$ & 0,08 & 0,07 \\
\hline PCS (MJ kg-1) & $-0,34$ & $-0,88$ & 0,22 & 0,43 & $-0,10$ & $-0,12$ & 0,08 & 0,07 \\
\hline PCL (MJ kg-1) & $-0,33$ & $-0,85$ & 0,18 & 0,35 & $-0,16$ & $-0,19$ & $-0,09$ & $-0,08$ \\
\hline DEL (GJ $\mathrm{m}^{-3}$ ) & $-0,32$ & $-0,83$ & 0,06 & 0,11 & $-0,21$ & $-0,24$ & $-0,33$ & $-0,31$ \\
\hline
\end{tabular}

C. fijo = carbono fijo; $\mathrm{PCl}$ = poder calorífico inferior; $\mathrm{PCS}$ = poder calorífico superior; $\mathrm{PCL}$ = poder calorífico liquido; $\mathrm{DEL}=$ densidad energética liquida 
El componente principal 1 se encuentra altamente correlacionada con los extraíbles, lignina, los poderes caloríficos, DTG, cenizas y residuo, por el cual puede ser interpretada como un índice de rendimiento energético. Cuando los valores de estas variables son mayores aumentará el valor numérico (score) del componente principal 1 (CP1) y más propicia será la biomasa para la producción de bioenergía. Analizando la segunda componente principal (CP2), se puede percibir que los mayores coeficientes en el módulo, se refiere a los porcentajes de hidrógeno, carbono y volátiles. El tercer componente (CP3), puede ser interpretado como el índice de polución ambiental y no como aprovechamiento energético de biomasas, ya que los mayores coeficientes de estos componentes son relativos a nitrógeno, cenizas y residuo.

Para la producción de bioenergía es deseable que la madera (biomasa) presente altos porcentajes de carbono e hidrogeno y bajos porcentajes de oxígeno y cenizas, en recurrencia de las relaciones existentes entre esas componentes elementales y el poder calorífico (Protásio,
Bufalino, Tonoli, Couto, Trugilho y Guimarães Júnior, 2011). Las variables más importantes para la clasificación y selección de biomasas para la generación de bioenergía presentan una mayor contribución en estos dos componentes. En este diagrama (Fig. 1), se observa la alta correlación de la lignina, extraíbles, carbono fijo, PCS, PCL y PCI, lo cual puede ser interpretado como un índice de rendimiento energético, ya que a mayores valores de estas variables mayor será el valor numérico (escores) de ese componente principal 1.

Los extractivos y la lignina ejercen un importante papel en el uso de la madera, ya que pueden influenciar en las propiedades físicas y energéticas (Vargas-Moreno et al., 2012), y dependiendo de la estabilidad térmica estas sustancias pueden ayudar al rendimiento de los pellets. Los resultados encontrados demuestran el potencial energético de estas dos biomasas (Paulownia y Miscanthus), y que puede resultar una opción para diversificar la matriz energética y también un aprovechamiento económico de estas biomasas.
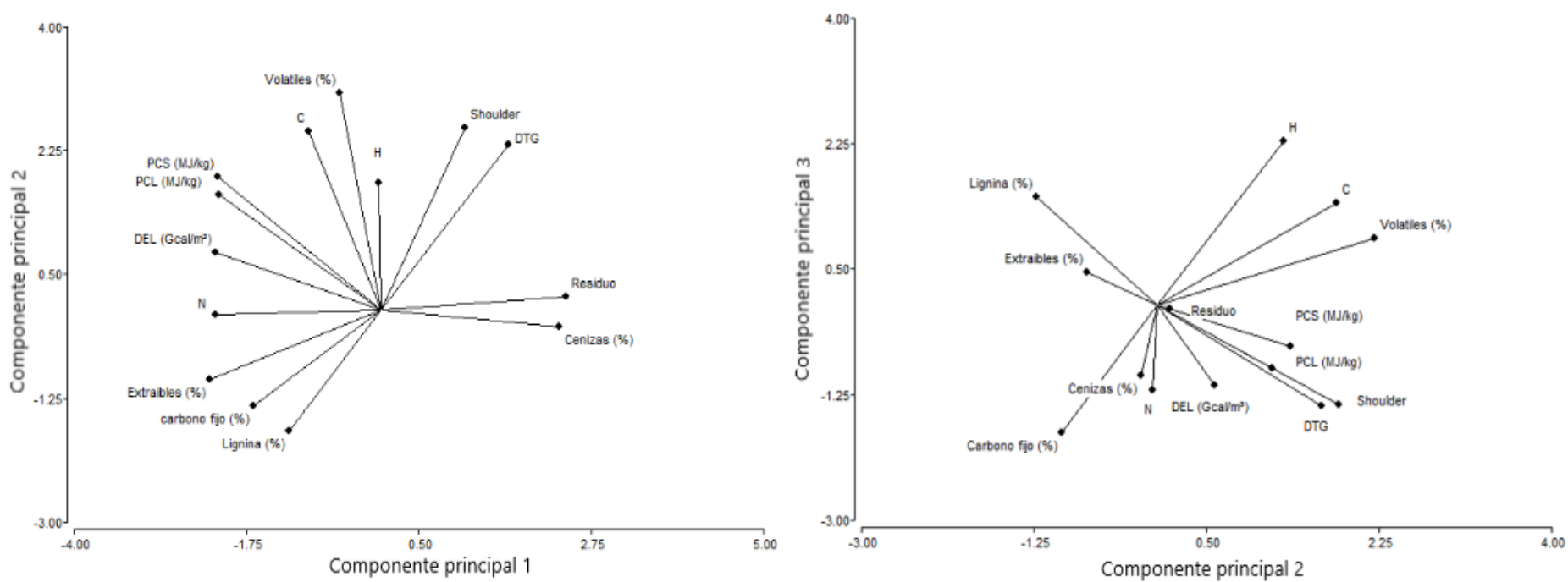

FIGURA 1. Diagrama de ordenación de variables originales y scores de las tres primeras componentes principales. 

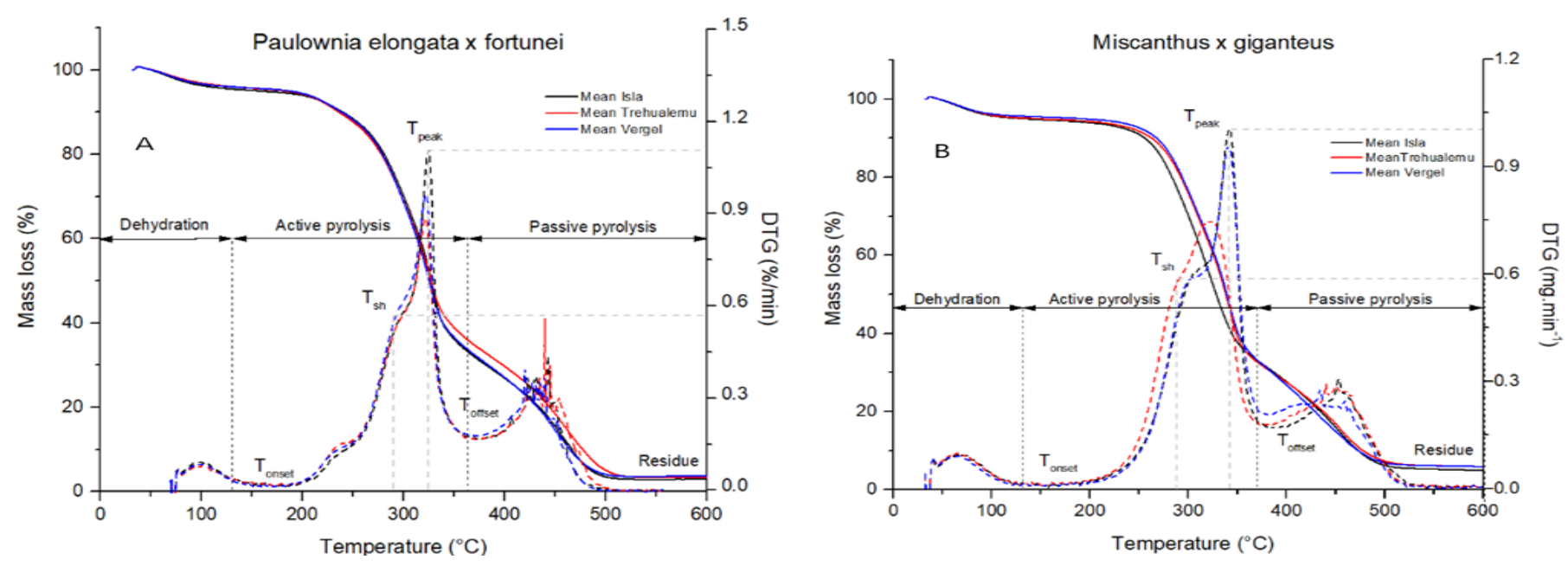

Figura 2. Curvas de TG (A) y DTG (B) de la Paulownia elongata $x$ fortunei

$T_{\text {onset }}=$ temperatura de ignición; $T_{\text {sh }}=$ temperatura máxima de degradación de la hemicelulose; $T_{\text {peak }}=$ máximo general de la pérdida de masa; $T$ offset $=$ valor final de la descomposición de la celulosa y zona de pirolisis activa.

\section{Características termogravimétricas de $P$. elongata $x$ fortunei y Miscanthus x giganteus}

Las curvas termogravimétricas (TG) y los diferenciales termogravimétricos (DTG) se muestran en la figura 2. La primera pérdida de peso ocurre en un rango de temperatura de $70{ }^{\circ} \mathrm{C}-150{ }^{\circ} \mathrm{C}$ para Paulownia y entre $40{ }^{\circ} \mathrm{C}-120{ }^{\circ} \mathrm{C}$ para Miscanthus, constituyendo una pérdida de peso de aproximadamente $10 \%$ en ambas especies. Esta pérdida de peso se atribuyó a la evaporación del agua, después comienza una segunda pérdida de peso en el rango de 290 ${ }^{\circ} \mathrm{C}-320{ }^{\circ} \mathrm{C}$ para Paulownia y entre $270{ }^{\circ} \mathrm{C}-310{ }^{\circ} \mathrm{C}$ para Miscanthus, se le atribuye a la exclusión de las hemicelulosas. La tercera pérdida (referente a la lignina), que se degrada en un amplio rango de temperatura $\left(350^{\circ} \mathrm{C}-600{ }^{\circ} \mathrm{C}\right)$, no muestra un pico característico como el de la hemicelulosa y celulosa (Poletto, Ornaghi Júnior y Zattera, 2014). Después de ocurrir estas tres pérdidas se puede eliminar hasta un 95\% del peso en ambas especies, estas pérdidas de peso se atribuyen principalmente a la celulosa (Sebio-Puñal, Naya, López-Beceiro, Tarrío-Saavedra, y Artiaga, 2012). Cada uno de estos componentes principales de las biomasas (hemicelulosa, celulosa y lignina), tienen su propio perfil de degradación térmica. El primer componente (hemicelulosa) son estructuras amorfas aleatorias y se pueden degradar fácilmente, el segundo componente es la celulosa que es polímero relativamente largo y son organizados (cristalinizado) y alta estabilidad térmica (Poletto et al., 2014). Por último, está la lignina que, de acuerdo con el mismo autor, posee tres tipos de unidades de bencenopropano fuertemente entre cruzadas, lo cual le otorga una alta estabilidad térmica y de degradación.

Finalmente ocurren unos picos estrechos en ambas biomasas en los rangos entre $430{ }^{\circ} \mathrm{C}-500^{\circ} \mathrm{C}$, que se relaciona con la descomposición y oxidación de la lignina residual y la combustión del carbono fijo formado entre la segunda y tercera fase. Estos picos corresponden a la combustión homogénea de gases liberados por la descomposición de la lignina y la combustión heterogénea de carbono sólido de acuerdo con Moon, Sung, Kim, Choi y Kim (2013). Estos resultados son similares a los encontrados por Chen, Wang, Meng, Wu, y Zhao, (2017) en Paulownia, a pesar de que en su investigación el residuo fue de $25 \%$ superior a los encontrados en este estudio que fue de 2,95\%. Respecto al Miscanthus, los valores aumentaron en relación a la Paulownia, tanto en el pico de hemicelulosa como en el de la celulosa. Estos resultados son semejantes a los de Wilk, Magdziarz, Gajek, Zajemska, Jayaraman y Gokalp, (2017), quienes estudiaron Miscanthus in natura $y$ torrefactado. Los mismos autores determinaron que los valores de hemicelulosa como de la 
celulosa son muy similares, salvo que las propiedades del material torrefactado se mejoran hacia el carbón lógicamente. Por lo tanto, estas especies presentan un perfil térmico considerable para un posible uso energético y ayudar a diversificar la gama de especies para la producción de energía.

Por otra parte, en la tabla 6 se muestran las temperaturas de degradación térmica de la biomasa y la masa residual a los $600{ }^{\circ} \mathrm{C}$ atribuible al carbón (char). Como podemos observar en ambas especies, la Paulownia tiene el peor comportamiento a medida que aumenta la temperatura, ya que tiene las temperaturas de inicio y final muy bajas; además, alcanza su mayor tasa de cambio de masa en la temperatura más baja (Tabla 6). Estos resultados son similares a los relatados por Villanueva, Proupín, Rodríguez-Añón, Fraga-Grueiro, Salgado y Barros(2011), aunque comparados con otros géneros como el eucalipto, pino o populus es bajo. Estas temperaturas son considerablemente más bajas que la del carbón vegetal ya que de acuerdo con Sahu, Sarkar, Chakraborty, y Adak (2010), la temperatura de ignición del carbón empieza a los $360{ }^{\circ} \mathrm{C}$. Pero la relevancia de estos resultados es que son similares a otras biomasas que hoy en día son utilizadas para la bioenergía, por lo tanto, son especies que pueden traer beneficios con sus plantíos. Por otro lado, en la tabla 7 se presenta un resumen de las clasificaciones de las biomasas analizadas en este estudio, de acuerdo con normas internacionales (alemanas), las cuales son unas de las más rigurosas en cuanto a calidad.

Una vez analizados los resultados de la Paulownia elongata $x$ fortunei, se puede evidenciar que el sitio que presentó los mejores resultados fue El Vergel, debido a que atendió la mayoría de los parámetros evaluados en este estudio. Al respecto, es importante destacar que los parámetros que no cumplen la norma se ubicaron muy cerca de los valores establecidos, como fue el azufre que no atendió los valores de la norma debido a la cercanía del sitio al mar, permitiendo con ello, recibir altas concentraciones de este componente. Este aspecto se debería tomar en consideración para posibles plantíos dendroenergéticos. Por lo tanto, se considera que el sitio El Vergel, a pesar de sus limitaciones de sitio, presentó los mejores resultados para plantar esta especie con fines energéticos.

TABLA 6. Temperatura de degradación térmica y su residuo (char) a $600{ }^{\circ} \mathrm{C}$ de Paulownia y Miscanthus

\begin{tabular}{|c|c|c|c|c|c|c|}
\hline Especie & Sitio & $T_{\text {in }}\left({ }^{\circ} \mathrm{C}\right)$ & $T_{\text {ter }}\left({ }^{\circ} \mathrm{C}\right)$ & $\begin{array}{c}T^{\circ} \text { Shoulder } \\
\left({ }^{\circ} \mathrm{C}\right)\end{array}$ & $\begin{array}{c}\text { Temperatura Peak } \\
\left({ }^{\circ} \mathrm{C}\right)\end{array}$ & $\begin{array}{l}\text { Residuo } \\
\text { (\%) }\end{array}$ \\
\hline \multirow{3}{*}{ Paulownia } & La Isla & & & $296,11^{( \pm 1)}$ & $329,00^{( \pm 1)}$ & $2,95^{( \pm 1)}$ \\
\hline & Trehualemu & $197.76^{( \pm 0,12)}$ & $377.76^{( \pm 0,12)}$ & $295,11^{( \pm 1)}$ & $325,67^{( \pm 1)}$ & $3,34^{( \pm 1)}$ \\
\hline & El Vergel & & & $295,44^{( \pm 1)}$ & $326,00^{( \pm 1)}$ & $3,70^{( \pm 1)}$ \\
\hline \multirow{3}{*}{ Miscanthus } & La Isla & & & $287,66^{( \pm 1)}$ & $323,00^{( \pm 1)}$ & $5,00^{( \pm 1)}$ \\
\hline & Trehualemu & $209.66^{( \pm 0,21)}$ & $379.18^{( \pm 0,21)}$ & $306,83^{( \pm 1)}$ & $341,33^{( \pm 1)}$ & $5,95^{( \pm 1)}$ \\
\hline & El Vergel & & & $304,49^{( \pm 1)}$ & $340,83^{( \pm 1)}$ & $5,94^{( \pm 1)}$ \\
\hline
\end{tabular}

$T_{\text {in }}=$ Temperatura inicial de la pirolisis; $T_{\text {ter }}=$ Temperatura final de la pirolisis 
TABLA 7. Clasificación de las biomasas de acuerdo con las normas de calidad.

\begin{tabular}{|c|c|c|c|c|c|c|c|c|c|}
\hline Normas & 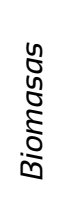 & $\stackrel{\circ}{\stackrel{\vdots}{n}}$ & $\begin{array}{l}\bar{D} \\
\frac{0}{0} \\
\frac{0}{4} \\
\frac{0}{0} \\
0\end{array}$ & 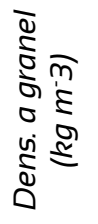 & 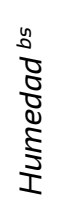 & ปั. & 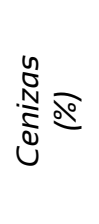 & 产す & 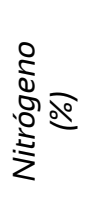 \\
\hline \multirow{3}{*}{$\begin{array}{c}\text { Biomasas maderables } \\
\text { EN 14961-2 } \\
\text { (DIN, 2011a) }\end{array}$} & \multirow{3}{*}{ 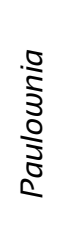 } & Trehua & $\mathrm{D} 1$ & $\checkmark$ & $\checkmark$ & $x$ & $\sqrt{ } \mathrm{B}$ & $x$ & $\checkmark A$ \\
\hline & & La Isla & $\mathrm{D} 1$ & $\checkmark$ & $\checkmark$ & $x$ & $\checkmark A$ & $x$ & $\checkmark A$ \\
\hline & & El Vergel & $\mathrm{D} 1$ & $\checkmark$ & $\checkmark$ & $x$ & $\checkmark A$ & $x$ & $\checkmark A$ \\
\hline \multirow{6}{*}{$\begin{array}{c}\text { Biomasas no maderables } \\
\text { EN 14961-6 } \\
\text { (DIN, 2012) }\end{array}$} & \multirow{6}{*}{ 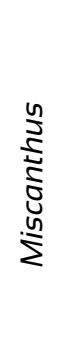 } & Trehua & $\mathrm{D} 1$ & $\checkmark$ & $\checkmark$ & $\sqrt{ } \mathrm{B}$ & $\checkmark A$ & $x$ & $\checkmark A$ \\
\hline & & Trehua & $\mathrm{D} 2$ & $\checkmark$ & $\checkmark$ & $\sqrt{ } B$ & $\checkmark A$ & $x$ & $\checkmark A$ \\
\hline & & La Isla & $\mathrm{D} 1$ & $\checkmark$ & $\checkmark$ & $\sqrt{ } B$ & $\sqrt{ } B$ & 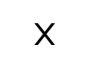 & $\checkmark A$ \\
\hline & & La Isla & $\mathrm{D} 2$ & $\checkmark$ & $\checkmark$ & $\checkmark A$ & $\sqrt{ } B$ & $x$ & $\checkmark A$ \\
\hline & & El Vergel & $\mathrm{D} 1$ & $\checkmark$ & $\checkmark$ & $\checkmark A$ & $\checkmark A$ & $x$ & $\checkmark A$ \\
\hline & & El Vergel & $\mathrm{D} 2$ & $\checkmark$ & $\checkmark$ & $\checkmark A$ & $\checkmark A$ & $x$ & $\checkmark A$ \\
\hline
\end{tabular}

$\checkmark$ : parámetro cumplido; $X$ = parámetro no cumplido; $A$ - B: clasificación de acuerdo con las normas.

Respecto al Miscanthus x giganteus sucede el mismo caso, los mejores valores se reportaron en El Vergel, destacando que la mayoría de los resultados son considerados como categoría A que corresponden a la clasificación de mejor calidad. Cabe destacar que para biomasas no maderables los parámetros son menos rigurosos. Los análisis de los parámetros energéticos a la biomasa de esta especie indican que creció bien en estas condiciones y puede ser plantada en diferentes sitios sin ningún problema para fines energéticos.

\section{CONCLUSIONES}

La especie Paulownia elongata $\times$ fortunei varió muy poco a sus propiedades químicas y energéticas entre los diferentes sitios, mostrando un buen nivel de adaptación. Los mejores resultados referentes a energía (lignina, extractivos, PCL y DEL) se registraron en El Vergel.

Respecto a la especie Miscanthus × giganteus, se puede decir que las características varían de acuerdo con el sitio y las densidades de los plantíos. Las condiciones (mediterráneas) del sitio El Vergel son ideales para el establecimiento de esta especie con fines energéticos.

Ambas biomasas (Paulownia y Miscanthus) presentan termogramas de combustión diferentes y en fases distintas, determinándose que el material lignocelulósico muestra aptitud para la producción directa de calor, debido a su baja temperatura de ignición.

$\mathrm{El}$ análisis de componentes principales género grupos homogéneos de las variables analizadas para identificar parámetros bioenergéticos, indicando que las biomasas evaluadas es fuente directa de bioenergía, esto por sus elevados valores caloríficos. En general, ambas especies se establecen bien luego se ser plantadas en los diferentes suelos y poseen un potencial energético interesante para ser utilizadas a una escala comercial.

\section{REFERENCIAS}

American Society for Testing and Materials [ASTM] (2007). D1762-84 Standard method for chemical analyses of wood charcoal.

Baxter, C., Darvell, I., Jones, M., Barraclough, T., Yates, E., \& Shield, I. (2014). Miscanthus combustion properties and variations with 
Miscanthus agronomy. Fuel, 117, 851-869. doi: 10.1016/j.fuel.2013.09.003.

Chen, L., Wang, S., Meng, H., Wu, Z., \& Zhao, J. (2017). Synergistic effect on thermal behavior and char morphology analysis during co-pyrolysis of paulownia wood blended with different plastics waste. Applied Thermal Engineering, 111, 834-846. doi: 10.1016/j.applthermaleng.2016.09.155.

Centro Nacional para la Innovación y Fomento de las Energías Sustentables [CIFES] (2016). Reportes Cifes: Energias renovables en el mercado eléctrico chileno. Santiago: Ministerio de Energía, Gobierno de Chile.

Delaye, M., \& Maia, F. (2015). Biochar a partir de biomassa de Miscanthus e Quebracho, produzido em forno tubular em sistema aberto e sob fluxo de nitrogênio. Embrapa Florestas-Comunicado Técnico (INFOTECA-E). Retrieved from https://www.infoteca.cnptia.embrapa.br/infoteca/bitstream/do c/1039706/1/ComunicadoTecnico3752015.pdf.

Demirbas, A. (2002). Relationships between heating value and lignin, moisture, ash and extractive contents of biomass fuels. Energy Exploration \& Exploitation, 20(1), 105-111. doi: 10.1260/014459802760170420.

Demirbaş, A. (2003). Relationships between heating value and lignin, fixed carbon, and volatile material contents of shells from biomass products. Energy Sources, 25(7), 629-635. doi: 10.1080/00908310390212336.

Deutsches Institut für Normung [DIN] (2010a). DIN EN 14774-1: Determination of moisture content - Oven dry method - Part 1: Total moisture - Reference method. Berlim: CEN.

Deutsches Institut für Normung [DIN] (2010b). DIN EN 14918: Determination of calorific value. Berlim: CEN.

Deutsches Institut für Normung [DIN] (2010c). DIN EN 15103: Determination of bulk density. Berlim: CEN.

Deutsches Institut für Normung [DIN] (2011a). DIN EN 14961-2: Solid biofuels - Fuel specifications and classes - Part 2: Wood pellets for non-industrial use. Berlim: CEN.

Deutsches Institut für Normung [DIN] (2011b) DIN EN 15104: Determination of total content of carbon, bydrogen and nitrogen Instrumental methods. Berlim: CEN.

Deutsches Institut für Normung [DIN] (2011c). DIN EN 15296: Conversion of analytical results from one basis to another. Berlim: CEN.

Deutsches Institut für Normung [DIN] (2012). DIN EN 14961-6: Solid biofuels - Fuel specifications and classes - Part 6: Non-woody pellets for nonindustrial use. Berlim: CEN.
El-Bassam, N. (1996). Renewable energy, potential energy crops for Europe and the Mediterranian Region. REU technical series 46. FAO Regional Office for Europe.

Facultad de Agronomía de la Universidad de Buenos Aires [FAUBA] (2012). Biomasa y energía. Recuperado de http:/ / ri.agro.uba.ar/cgibin/library.cgi-about-miscanthus.

Fernández-Puratich, H., Oliver-Villanueva, J., Mireya, V., Salvador, V., $\&$ Nuria, A. (2014). Desarrollo de pellets a partir de tres especies leñosas bajo condiciones mediterráneas. Madera y Bosques, 20(3), 97-111. doi: 10.21829/myb.2014.203155

Gillespie, D., Everard, D., Fagan, C., \& Mcdonnell, P. (2013). Prediction of quality parameters of biomass pellets from proximate and ultimate analysis. Fuel, 111, 771-777.

Guofeng, S. (2014). Emission factors of carbonaceous particulate matter and polycyclic aromatic bydrocarbons from residential solid fuel combustions. Recuperado de https://link.springer.com/book/10.1007/978-3642-39762-2\#about.

Ghetti, P. (1986). DTG combustion behaviour of coal: Correlations with proximate and ultimate analysis data. Fuel, 65(5), 636-639.

Hua, Z., Ching-Ju C., Xin-Yu L., \& Yao Gao Z. 1986. Cultivation and Utilization, Academy of Forestry, ANFBS and IDRC, Beijing, China.

Kalaycioglu, H., Deniz, I., \& Hiziroglu, S. (2005). Some of the properties of particleboard made from paulownia. Journal of Wood Science, 51(4), 410-414. doi: 10.1007/s10086-004-0665-8.

Kasamaki P. (2007). La Paulownia sp. como base de los cultivos energéticos. Recuperado de http:// www.vicedex.com/pdf/paulownia.pdf.

Lewandowski, I., Clifton-Brown, J., Scurlock, J., \& Huisman, W. (2000). Miscanthus: European experience with a novel energy crop. Biomass and Bioenergy, 19, 209-227.

Lino, J., Fernando, A., Barbosa, B., Boléo, S., Costa, J., Duarte, M., \& Mendes, B. (2014). Phytoremediation of $\mathrm{Cd}$ and Ni contaminated wastewaters by Miscanthus. En C. Hoffmann, D. Baxter, K. Maniatis, A. Grassi, \& P. Helm, (Eds.) Proceedings of the 22nd European biomass conference and exbibition, setting the course for a biobased economy (pp. 303 - 307). ETA-Renewable Energies.

López, F., Pérez, A., Zamudio, M., De Alva, E., \& García, J. C. (2012). Paulownia as raw material for solid biofuel and cellulose pulp. Biomass and Bioenergy, 45, 77-86. doi: 10.1016/j.biombioe.2012.05.010.

Meehan, G., Finnan, M., \& Donnell, M. (2013). The effect of harvest date and harvest method on the combustion characteristics of Miscanthus $\propto$ giganteus. GCB Bioenergy, 5(5), 487-496. doi: 10.1111/gcbb.12003. 
Mendonça, R. T., Jara, J. F., González, V., Elissetche, J. P., \& Freer, J. (2008). Evaluation of the white-rot fungi Ganoderma australe and Ceriporiopsis subvermispora in biotechnological applications. Journal of Industrial Microbiology and Biotechnology, 35(11), 1323-1330. doi: 10.1007/s10295-008-0414-x.

Moon, C., Sung, Y., Ahn, S., Kim, T., Choi, G., \& Kim, D. 2013. Effect of blending ratio on combustion performance in blends of biomass and coals of different ranks. Experimental Thermal and Fluid Science, 4, 232- 240.

McKendry, P. (2002). Energy production from biomass (part 1): Overview of biomass. Bioresource Technology, 83(1), 37-46. doi: 10.1016/S0960-8524(01)00118-3

Muñoz, F. \& Cancino, J. (2014a). Antecedentes de Miscanthus x giganteus para la producción de bioenergía. In, Facultad de Ciencias Forestales, Universidad de Concepción. Recuperado de http://www2.udec.cl/ fmunoz/Libro_Miscanthus.pdf.

Muñoz, F. \& Cancino, J. (2014b). Antecedentes de Paulownia elongata x fortunei para la producción de bioenergía. Facultad de Ciencias Forestales, Universidad de Concepción. Recuperado de http://www2.udec.cl/ fmunoz/Libro_Paulownia.pdf.

Muñoz, F., Cancino, J., Rodríguez, R., \& Olave, R. (2018). Evaluación de crecimiento, rendimiento y calorimetría de biomasa de Miscanthus $\times$ giganteus (Poaceae) establecido en el centro-sur de Chile. Revista de La Facultad de Ciencias Agrarias, 50(1), 1853-8665.

Obernberger, I., Brunner, T., \& Barnthaler, G. (2006). Chemical properties of solid biofuels - significance and impact. Biomass and Bioenergy, 30(11), 973-982.

Obernberger, I. \& Thek, G. (2010). The pellet handbook: The production and thermal utilisation of pellets. London: Earthscan.

Paula, R., Trugilho, F., Napoli, A., \& Bianchi, M. (2011). Characterization of residues from plant biomass for use in energy generation. Cerne, 7(2), 237-246.

Pereira, C. (2014). Propriedades de pellets de diferentes biomassas para fins energéticos. Dissertação doutoral. Universidade Federal de vicosa, Minas Gerais, Brasil.

Pinto, A. (2015). Avaliação da biomassa de Paulownia enquanto combustível na forma peletizada. Dissertação de mestrado, Instituto politécnico de Viseu, Portugal.

Płażek, A. \& Dubert, F. (2010). Improvement of Medium for Miscanthus $x$ Giganteus Callus Induction and Plant Regeneration. Acta Biologica Cracoviensia Series Botanica, 52(1), 105-110. doi: 10.2478/v10182010-0013-9.

Poddar, S., Kamruzzaman, M., Sujan, A., Hossain, M., Jamal, S., \& Gafur, A. (2014). Effect of compression pressure on lignocellulosic biomass pellet to improve fuel properties: Higher heating value. Fuel, 131, 43-48. doi: 10.1016/j.fuel.2014.04.061.

Poletto, M., Ornaghi Júnior, L., \& Zattera, J. (2014). Native cellulose: Structure, characterization and thermal properties. Materials, 7(9), 6105-6119. doi: 10.3390/ma7096105.

Protásio, T., Bufalino, L., Tonoli, D., Couto, M., Trugilho, F., \& Guimarães Júnior, M. (2011). Relação entre o poder calorífico superior e os componentes elementares e minerais da biomassa vegetal. Pesquisa Florestal Brasileira, 31(66), 113-122. doi: 10.4336/2011.pfb.31.66.113.

Roncucci, N., Nassi O Di Nasso, N., Bonari, E., \& Ragaglini, G. (2015). Influence of soil texture and crop management on the productivity of miscanthus (Miscanthus $\times$ giganteus Greef et Deu.) in the Mediterranean. GCB Bioenergy, 7(5), 998-1008. doi: 10.1111/gcbb.12202.

Rosa, A., Hortal, J., Vidal, T., Colom, J., \& Torres, A. (2004). Caracterización de Kenaf y Miscanthus sinensis para la fabricación de papel. Revista de química teórica y aplicada, 61, 12 - 24.

Santiago-Ortega, M., Honorato-Salazar, J., Quero-Carrillo, A., Hernandez-Garay, A., López-Castañeda, C., \& López-Guerrero, I. (2016). Biomasa de Urochloa brizantha cv. Toledo como materia prima para la producción de etanol. Agrociencia, 50, 711 726.

Saldarriaga, F., Pablos, A., Amutio, M., Aguado, R., Olazar, M. (2013). Determination of moisture content, proximate and lignocellulosic analysis of biomass by thermogravimetric analysis. Recuperado de http://www.aeipro.com/files/congresos/2013logronio/CIDIP 2013_1001_1010.4025.pdf.

Sahu, G., Sarkar, P., Chakraborty, N., \& Adak, K. (2010). Thermogravimetric assessment of combustion characteristics of blends of a coal with different biomass chars. Fuel Processing Technology, 91(3), 369-378. doi: 10.1016/j.fuproc.2009.12.001.

Sebio-Puñal, T., Naya, S., López-Beceiro, J., Tarrío-Saavedra, J., \& Artiaga, R. (2012). Thermogravimetric analysis of wood, holocellulose, and lignin from five wood species. Journal of Thermal Analysis and Calorimetry, 109(3), 1163-1167. doi: 10.1007/s10973011-2133-1.

Scally, L., Hodkinson, T., \& Jones, M. (2007). Origins and taxonomy of Miscanthus. En M. Jones \& M. Walsh (Eds.). Miscanthus for energy and fibre. London, UK: Earthscan.

Technical Association of the Pulp and Paper Industry [TAPPI] (1985). TAPPI T $257 \mathrm{~cm}-85$. Sampling and preparing wood for analysis. Atlanta: TAPPI. 
Technical Association of the Pulp and Paper Industry [TAPPI] (1997).

TAPPI T $204 \mathrm{~cm}-97$. Solvent extractives of wood and pulp. Atlanta: TAPPI, 1997.

Tolosana, E. (2009). Manual técnico para el aprovechamiento y elaboración de biomasa forestal. Madrid. FUCOVASA/ Mundi-prensa.

Vargas-moreno, J. M., Callejón-ferre, A. J., Pérez-alonso, J., \& Velázquez-martí, B. (2012). A review of the mathematical models for predicting the heating value of biomass materials. Renewable and Sustainable Energy Reviews, 16(5), 3065-3083. doi: 10.1016/j.rser.2012.02.054.

Velázquez-martí, B., Sajdak, M., López-cortés, I., \& Callejón-ferre, J. (2014). Wood characterization for energy application proceeding from pruning Morus alba L., Platanus bispanica Münchh. and Sophora japonica L . in urban areas. Renewable Energy, 62, 478-483. doi: 10.1016/j.renene.2013.08.010.

Villanueva, M., Proupín, J., Rodríguez-Añón, A., Fraga-Grueiro, L., Salgado, J., \& Barros, N. (2011). Energetic characterization of forest biomass by calorimetry and thermal analysis. Journal of Thermal Analysis and Calorimetry, 104(1), 61-67. doi: 10.1007/s10973-010-1177-y.

Wang, Q., \& Shogren, F. (1992). Characteristics of the crop-paulownia system in China. Agriculture. Ecosystems and Environment 39, 145152.
Wilk, M., Magdziarz, A., Gajek, M., Zajemska, M., Jayaraman, K., \& Gokalp, I. (2017). Combustion and kinetic parameters estimation of torrefied pine, acacia and Miscanthus giganteus using experimental and modelling techniques. Bioresource Technology, 243, 304-314. doi: 10.1016/j.biortech.2017.06.116.

Zattera, J., Bianchi, O., Zeni, M., \& Ferreira, C. (2005). Caracterização de resíduos de copolímeros de etileno-acetato de vinila - EVA. Polimeros: Ciência e Tecnologia, 15(1), 73-78. doi: 10.1590/S010414282005000100016

Manuscrito recibido el 21 de diciembre de 2018

Aceptado el 25 de julio de 2019

Publicado el 11 de abril de 2020

Este documento se debe citar como:

Pegoretti, H., Muñoz, F., Teixeira. R., Sáez, K., Segura, C., \& Olave, R. (2020). Potencial energético de clones de Paulownia y Miscanthus para la producción de energía en Chile. Madera y Bosques, 26(1), e2611916. doi: 10.21829/myb.2020.2611916.

Madera y Bosques por Instituto de Ecología, A.C. se distribuye bajo una Licencia Creative Commons Atribución-NoComercialCompartirlgual 4.0 Internacional. 